\title{
Consumo precoce de alimentos não recomendados por lactentes do sul do Brasil
}

\author{
Early consumption of non-recommended foods among southern Brazilian infants
}

\author{
Márcia Regina Vitolo, Maria Laura da Costa Louzada², Gabriela Possa², \\ Gisele Ane Bortolini ${ }^{3}$
}

\begin{abstract}
RESUMO
Objetivo: Avaliar o consumo de alimentos não recomendados em crianças assistidas no âmbito da Atenção Primária à Saúde no município de Porto Alegre, RS.

Materiais e Métodos: A amostra compreendeu crianças residentes no território de cobertura de vinte Unidades de Saúde. As mães das crianças de 6 a 9 meses de idade responderam a um questionário fechado, em que ela confirmava ou não se a criança já tinha consumido determinados alimentos. Posteriormente, na idade de 12 a 15 meses, foi realizada nova investigação quanto ao consumo dos mesmos alimentos no último mês.

Resultados: Refrigerante, bala ou pirulito e biscoito recheado foram consumidos por, aproximadamente, $40 \%$ das crianças na idade de 6 a 9 meses e $80 \%$ na idade de 12 a 15 meses. A prevalência de consumo de açúcar de adição, queijo petit suisse e gelatina foi superior a $70 \%$ nos dois momentos. Alimentos fritos e chocolate foram consumidos por mais de $80 \%$ na idade de 12 a 15 meses.

Conclusão: Os resultados mostram elevada prevalência de consumo de alimentos não recomendados para crianças menores de dois anos, enfatizando a necessidade de sensibilização, formação e preparo dos profissionais da Atenção Primária à Saúde para a realização de aconselhamento dietético.
\end{abstract}

Palavras-chave: lactente; consumo de alimentos; atenção primária à saúde.

\begin{abstract}
Objective: To evaluate the consumption of non-recommended foods among children under the primary care assistance service in the city of Porto Alegre, RS.

Materials and Methods: The sample included children living in the territory of twenty health care centers. The mothers of the 6 to 9 months old children answered to a structured questionnaire confirming if the children had already eaten some specific foods. Afterwards, at 12 to 15 months, another investigation related to the children's food consumption during the last month was conducted.

Results: Soft drink, candies and cookies were consumed by, approximately, 40\% of the children at 6 to 9 months and $80 \%$ at 12 to 15 months. The consumption prevalence of table sugar, petit suisse and jelly was above $70 \%$ at both moments. Fried foods and chocolate were consumed by more than $80 \%$ of the children at 12 to 15 months old.

Conclusion: The results of this study show a high consumption prevalence of non-recommended foods among children under two years old, emphasizing the importance of improving dietary counseling skills of primary care workers.
\end{abstract}

Keywords: infant; food consumption; primary health care.

\footnotetext{
${ }^{1}$ Nutricionista. Doutora em Ciência Biológicas. Departamento de Nutrição, Universidade Federal de Ciências da Saúde de Porto Alegre. ${ }^{2}$ Nutricionista. Mestre em Ciências da Saúde. Programa de Pós-graduação em Ciências da Saúde, Universidade Federal de Ciências da Saúde de Porto Alegre.

${ }^{3}$ Nutricionista. Mestre em Ciências da Saúde. Coordenação Geral de Alimentação e Nutrição, Departamento de Atenção Básica, Secretaria de Atenção à Saúde, Ministério da Saúde.
} 


\section{INTRODUÇÃO}

Nos dois ou três primeiros anos de vida, a qualidade dos alimentos ofertados para a criança é fundamental para a formação dos hábitos alimentares futuros. Além disso, o consumo de alimentos ricos em açúcar e sal vão prejudicar a ingestão de alimentos de melhor densidade nutricional ${ }^{1}$. O consumo de alimentos altamente energéticos, no entanto, é frequente em populações de baixa condição socioeconômica, devido ao baixo custo dos mesmos em comparação com alimentos de alta densidade de nutrientes ${ }^{2}$.

Os "Dez passos para alimentação saudável para crianças brasileiras menores de dois anos" são recomendações descritas em manual técnico para subsidiar os profissionais da saúde, em especial aqueles da Atenção Primária à Saúde, na promoção de práticas alimentares saudáveis para a criança pequena ${ }^{3}$. O passo oito consiste em "evitar açúcar, café, enlatados, frituras, refrigerantes, balas, salgadinho e outras guloseimas, nos primeiros anos de vida, e usar sal com moderação", com a finalidade de diminuir os riscos do desenvolvimento de deficiências de micronutrientes e excesso de peso associados aos hábitos alimentares inadequados.

O objetivo deste estudo foi avaliar a prevalência de consumo de alimentos não recomendados para crianças menores de dois anos em população atendida no âmbito da Atenção Primária à Saúde (APS) no município de Porto Alegre.

\section{MATERIAIS E MÉTODOS}

A amostra foi composta de crianças residentes no território de vinte Unidades de Saúde do município de Porto Alegre, RS, as quais participam de projeto de pesquisa maior - ensaio de campo randomizado por conglomerados - realizado entre dezembro de 2008 e fevereiro de 2010. Gestantes que realizaram pré-natal nas Unidades de Saúde do estudo foram convidadas a participarem da pesquisa. Após o consentimento, responderam a questionário sobre dados socioeconômicos e demográficos e seus filhos foram avaliados durante o primeiro ano de vida.

Os dados apresentados foram gerados a partir de questionário aplicado às mães e/ou cuidadores dos lactentes, os quais foram entrevistados quanto ao consumo, pelas crianças, dos seguintes alimentos e bebidas: açúcar de adição, mel, suco artificial (pó), refrigerante, gelatina, queijo petit suisse, bala/pirulito, bolacha recheada, chocolate, salgadinho tipo chips, alimentos fritos (batata, aipim, pastel, bolo de carne) e embutidos (presunto, mortadela, salame, salsicha). A seleção desses alimentos levou em consideração a presença de elevada concentração de açúcar e de sal na composição nutricional desses de acordo com o oitavo passo das diretrizes Brasileiras para alimentação saudável para crianças menores de dois anos ${ }^{3}$. As entrevistas foram realizadas em duas visitas domiciliares: a primeira quando as crianças tinham idades entre 6 e 9 meses, em que a mães referiram se seu filho já tinha consumido um daqueles alimentos listados anteriormente; e na segunda entrevista, que ocorreu quando as crianças tinham idade entre 12 e 15 meses. Nesta entrevista a investigação se referiu ao consumo dos alimentos não recomendados no mês anterior à entrevista. Os resultados do consumo alimentar foram obtidos considerando o fato de a criança já ter consumido, alguma vez, o alimento referido pelo entrevistador em perguntas fechadas.

Os dados foram tabulados no software Statistical Package for Social Sciences (SPSS) versão 16.0 e as análises de frequência efetuadas no mesmo programa. O projeto foi aprovado pelo Comitê de Ética da Universidade Federal de Ciências da Saúde de Porto Alegre (UFCSPA) e foi registrado no ClinicalTrials.gov sob o identificador NCT00635453.

\section{RESULTADOS}

Avaliaram-se 619 crianças aos 6 a 9 meses e 532 aos 12 a 15 meses. As famílias que participaram do estudo apresentaram as seguintes características socioeconômicas e demográficas: $21,2 \%$ das mães tinham idade inferior a 20 anos, 31,6\% apresentavam renda familiar mensal igual ou menor que 600 reais, $29,9 \%$ das mães se declararam donas de casa e $31 \%$ das mães tinham menos de 8 anos de escolaridade. $\mathrm{Na}$ tabela 1, observam-se os percentuais de crianças que já tinham consumido alimentos não recomendados para a faixa etária estudada. 
TABELA 1 - Prevalência de consumo de alimentos e bebidas não recomendados em crianças residentes no território de vinte unidades de saúde de porto alegre (2008-2010).

\begin{tabular}{lcccc}
\hline Alimentos/Bebidas & $\begin{array}{c}\mathbf{6} \text { a 9 meses } \\
\text { de idade } \\
(\mathbf{n = 6 1 9})\end{array}$ & $\begin{array}{c}\mathbf{1 2} \text { a 15 meses } \\
\text { de idade } \\
(\mathbf{n}=532)\end{array}$ \\
\hline Açúcar de adição & $\mathbf{n}$ & $\%$ & $\mathbf{n}$ & $\%$ \\
Queijo petit suisse & 459 & 74,2 & 497 & 94,7 \\
Gelatina & 451 & 73,0 & 501 & 95,2 \\
Refrigerante & 269 & 43,5 & 458 & 87,1 \\
Bala ou pirulito & 261 & 42,2 & 462 & 87,7 \\
Biscoito recheado & 235 & 38,0 & 464 & 88,2 \\
Chocolate & 188 & 30.4 & 458 & 87,1 \\
Mel & 154 & 25,1 & 236 & 44,9 \\
Suco artificial (pó) & 144 & 23,3 & 374 & 70,8 \\
Salgadinho tipo chips & 122 & 19,7 & 381 & 72,4 \\
Alimentos fritos & 106 & 17,1 & 433 & 82,0 \\
Embutidos† & 53 & 8,6 & 367 & 69,6 \\
\hline
\end{tabular}

${ }^{*}$ Batata frita, aipim frito, pastel frito, bolo de carne frito. †Presunto, mortadela, salame, salsicha.

\section{DISCUSSÃO}

O percentual elevado de crianças menores dois anos que consumiram alimentos de baixo valor nutricional e alta densidade energética é um resultado que tem impacto na saúde dessas crianças, já que o consumo desses alimentos é preocupante no primeiro ano de vida, período em que as crianças deveriam receber alimentação complementar adequada com o intuito de formar hábitos alimentares saudáveis.

A transição nutricional, também evidenciada no Brasil, caracteriza-se pela presença concomitante da deficiência de micronutrientes, excesso de peso e outras doenças na mesma comunidade e muitas vezes no mesmo domicílio ${ }^{4}$. No Brasil, aproximadamente $40 \%$ da população brasileira se encontra em situação de insegurança alimentar, ou seja, possuem seu direito humano à alimentação adequada violado e, dessa forma, não possuem acesso contínuo à alimentação em quantidade e/ou qualidade ${ }^{5}$. Como conseqüência desse padrão de consumo, 3,3 milhões de crianças brasileiras menores de cinco anos apresentam anemia e 2,7 milhões hipovitaminose $A^{6}$. O excesso de peso e a obesidade infantil, também já podem ser considerados importantes problemas de saúde pública. Dados recentes, de pesquisas nacionais, mostraram que a prevalência de excesso de peso em crianças menores de cinco anos é de aproximadamente $7,6 \%$ e de $33,5 \%$ nas crianças em idade escolar ${ }^{7}$.

Levando em consideração a alta prevalência de excesso de peso em crianças na idade escolar é possível afirmar que os primeiros anos de vida podem ser fundamentais para a reversão desse quadro, uma vez que as preferências alimentares e padrões dietéticos estabelecem-se precocemente e há evidências de que os alimentos que a criança recebe nos primeiros anos de vida podem se prolongar até a vida adulta ${ }^{1}$.

Hábitos alimentares inadequados constituem-se em problema de saúde pública, tendo em vista que o consumo de alimentos não apropriados para a idade pode estar associado à presença de deficiências de micronutrientes, excesso de peso, alergias alimentares, caries e botulismo na infância, além do desenvolvimento das doenças crônicas não transmissíveis na vida adulta ${ }^{8-13}$

Esse estudo evidencia o inadequado consumo alimentar em crianças residentes no território de atuação de vinte Unidades de saúde do município de Porto Alegre. A promoção da mudança de padrões dietéticos é complexa e ainda mais difícil na vida adulta, - que torna particularmente importante 0 desenvolvimento de ações precoces e que promovam hábitos alimentares saudáveis por toda a vida. No atual modelo de atenção à saúde adotado no Brasil, a APS é o espaço preferencial para o desenvolvimento de ações de caráter individual e coletivo, voltadas à promoção da saúde e em especial à alimentação saudável. Vários estudos já demonstraram o impacto do modelo assistencial na saúde das crianças ${ }^{14}$. A Estratégia Saúde da Família é o modelo que ordena as ações da APS no Brasil, e atualmente conta com mais de 30 mil equipes, com cobertura de $52,3 \%$ da população brasileira. No entanto, para a reversão do atual cenário epidemiológico, de consumo de alimentos não recomendados na infância e excesso de peso em todo 
ciclo da vida, faz-se necessário o investimento em sensibilização, formação e preparo dos profissionais, que irão atuar e que são atuantes na APS, para que esses se tornem promotores da alimentação saudável.

\section{REFERÊNCIAS}

1. Skinner JD, Carruth BR, Wendy B, Ziegler PJ. Children's food preferences: a longitudinal analysis. J Am Diet Assoc. 2002;102(11):1638-47.

2. Drewnowski A, Specter SE. Poverty and obesity: The role of energy density and energy costs. Am J Clin Nutr. 2004;79(1):6-16

3. Ministério da Saúde. Dez passos para uma alimentação saudável: Guia alimentar para crianças menores de dois anos. Brasília: Ministério da Saúde; 2002.

4. Popkin BM. The nutrition transition and obesity in the developing world. J Nutr. 2001;131(Suppl 3):871-3.

5. Instituto Brasileiro de Geografia e Estatística. Pesquisa nacional por amostra de domicílios - PNAD 2004. Rio de Janeiro: Instituto Brasileiro de Geografia e Estatística; 2006.

6. Ministério da Saúde. Pesquisa nacional de demografia e saúde da criança e da mulher. Brasília: Ministério da Saúde; 2008.

7. Instituto Brasileiro de Geografia e Estatística. Pesquisa de orçamentos familiares 2008-2009. Rio de Janeiro: Instituto Brasileiro de Geografia e Estatística; 2010.

8. Kranz S, Smiciklas-Wright $H$, Siega-Riz AM, Mitchell D. Adverse effect of high added sugar consumption on dietary intake in american preschoolers. J Pediatr. 2005;146(1):105-11.
9. Ludwig DS, Peterson KE, Gortmaker SL. Relation between consumption of sugar-sweetened drinks and childhood obesity: A prospective, observational analysis. Lancet. 2001;357(9255):505-8.

10. Feldens CA, Giugliani ER, Vigo Á, Vítolo MR. Early feeding practices and severe early childhood caries in four-year-old children from southern Brazil: a birth cohort study. Caries Res. 2010;44(5):445-52.

11. Pac, S, McMahon, K, Ripple, M, Reidy K, Ziegler, $\mathrm{P}$, Myersa, E. Development of the Start Healthy Feeding Guidelines for infants and toddlers. JADA 2004;104(3): 455-67.

12. Dewey, KG. Approaches for improving complementary feeding of inants and young children. Geneva: World Health Organization; 2000.

13. Pereira MA, Kartashov Al, Ebbeling CB, Van Horn L, Slattery ML, Jacobs DR Jr, Ludwig DS. Fast-food habits, weight gain, and insulin resistance (the CARDIA study): 15-year prospective analysis. Lancet. 2005;365(9453):3642.

14. Macinko J, Marinho de Souza Mde F, Guanais FC, da Silva Simoes CC. Going to scale with community-based primary care: An analysis of the family health program and infant mortality in brazil, 1999-2004. Soc Sci Med. 2007;65:2070-80.

\section{Endereço para correspondência:}

Márcia Regina Vitolo

Rua Sarmento Leite 245, sala 208

Porto Alegre/RS - CEP 90050-170

Telefone: + 555133038830

Email: vitolo@ufcspa.edu.br 